\title{
On a set of convergent solutions for a system of second order linear difference equations
}

\author{
By \\ Masahiro Iwano* and Kenjiro Окuво
}

In this paper, we will show the existence of $2 n$ convergent solutions of a system of the second order difference equations

$$
\left(\rho+m-A_{0}\right) G(m)=A_{1} G(m-1)+A_{2} G(m-2)
$$

which are suitable for the study of the connection problem for the system of linear ordinary differential equations

$$
t \frac{d X}{d t}=\left(A_{0}+A_{1} t+A_{2} t^{2}\right) X
$$

The problem was proposed and formulated by the junior author, and the crucial point of the convergence proof was done by the senior author.

In (2), $A_{j}(j=0,1,2)$ are $n$ by $n$ constant matrices and $X$ is an $n$-dimensional vector, and $t$ is a complex variable. The system has 2 singular points in the closed complex plane; one at the origin, which is a regular singular point, the other at infinity which is an irregular singular point. We will assume the eigen-values $\lambda_{1}, \lambda_{2}, \cdots, \lambda_{n}$ of the matrix $A_{2}$ are mutually distinct, and they satisfy the following pentagonal condition

$$
\left|\lambda_{j}-\lambda_{k}\right|>|>| \lambda_{k} \mid>0 \quad(j \neq k)
$$

and without loss of generality, we will suppose that $A_{2}$ is in the diagonal form,

* Mathematics Research Center, University of Wisconsin, and Department of Mathematics, Tokyo Metropolitan University.

Received November 16, 1965. 


$$
A_{2}=\left(\begin{array}{lllll}
\lambda_{1} & & & \\
& \lambda_{2} & & 0 \\
& & \cdot & \\
0 & & \cdot & \\
& & & \\
& & & \lambda_{n}
\end{array}\right)
$$

Let $\rho$ be an eigenvalue of the matrix $A_{0}$, which has no integral difference with the other eigenvalues of $A_{0}$, then there is a convergent solution of the form

$$
X(t)=t^{\rho} \sum_{m=0}^{\infty} G(m) t^{m}
$$

with vector coefficients $G(m)$. If we substitute (4) into (2), we have a system of difference equations (1) to be satisfied by $G(m)$.

Our general plan is to investigate the behavior at infinity of the solution (4), by studying the asymptotic behavior of $G(m)$ for large values of $m$. But the initial conditions for $G(m)$

$$
\left\{\begin{array}{l}
\left(\rho-A_{0}\right) G(0)=0 \\
\left(\rho+1-A_{0}\right) G(1)=A_{1} G(0)
\end{array}\right.
$$

are given at $m=0$. So we must find an expression for $G(m)$ suitable for study at both $m=0$ and $m=\infty$. To that end, we use the formal solutions of (2) at $t=\infty$;

(6) $\quad X^{k}(t)=\exp \left(\left(\lambda_{k} t^{2} / 2+\mu_{k} t\right) t^{\sigma_{k}} \sum_{s=0}^{\infty} H^{k}(s) t^{-s} \quad(k=1,2, \cdots, n)\right.$

where the coefficient vectors $H^{k}(s)(k=1,2, \cdots, n)$ satisfy

$$
\begin{aligned}
& \left(s-\sigma_{k}+A_{0}\right) H^{k}(s)=\left(\mu_{k}-A_{1}\right) H^{k}(s+1)+\left(\lambda^{k}-A_{2}\right) H^{k}(s+2) \\
& \left(\lambda_{k}-A_{2}\right) H^{k}(0)=0, \quad\left(\lambda_{k}-A_{2}\right) H^{k}(1)+\left(\mu_{k}-A_{1}\right) H^{k}(0)=0 .
\end{aligned}
$$

We remark, that for second of the initial conditions to be satisfied, it is necessary that

$\mu_{k}=$ the $k$-th diagonal element of the matrix $A_{1}$.

Following the results of the paper [1] and [2], we define $g^{k}(m)$ to be a solution of the following single difference equation 
On a set of convergent solutions for a system...

$$
\left(\rho+m-\sigma_{k}\right) g^{k}(m)=\mu_{k} g^{k}(m-1)+\lambda_{k} g^{k}(m-2) .
$$

Proposition 1. Formal series

$$
G^{k}(m)=\sum_{s=0}^{\infty} H^{k}(s) g^{k}(m+s) \quad(k=1,2, \cdots, n)
$$

are formal solutions of the system (1).

(Proof.) We have

$$
\begin{aligned}
\left(\rho+m-A_{0}\right) \sum_{s=0}^{\infty} H^{k}(s) g^{k}(m+s) & =\sum_{s=0}^{\infty}\left[\left(\rho+m+s-\sigma_{k}\right)\right. \\
& \left.+\left(\sigma_{k}-s-A_{0}\right)\right] H^{k}(s) g^{k}(m+s) \\
=\sum_{s=0}^{\infty} H^{k}(s)\left[\mu_{k} g^{k}(m+s-1)+\right. & \left.\lambda_{k} g^{k}(m+s-2)\right] \\
+ & +\sum_{s=0}^{\infty}\left(\sigma_{k}-s-A_{0}\right) H^{k}(s) g^{k}(m+s)
\end{aligned}
$$

by definition (8), then we use (7) to have

$$
\begin{aligned}
& =A_{1} G^{k}(m-1)+A_{2} G^{k}(m-2)+\left[\left(\mu_{k}-A_{1}\right) H^{k}(1)\right] g^{k}(m-1) \\
& +\left(\lambda_{k}-A_{2}\right) H^{k}(0) g^{k}(m-2) \\
& =A_{1} G^{k}(m-1)+A_{2} G^{k}(m-2) .
\end{aligned}
$$

To prove the convergence of the series $G^{k}(m)$ and series such as

$$
\sum_{s=0}^{\infty} s H^{k}(m+s),
$$

we will define formal power series $F^{k}(m, \varepsilon)$ in complex variable $\varepsilon$ by

$$
F^{k}(m, \varepsilon)=\varepsilon^{\rho+m-\sigma_{k}} \sum_{s=0}^{\infty} H^{k}(s) g^{k}(m+s) \varepsilon^{s} \quad(k=1,2, \cdots, n) .
$$

We start by finding a system of differential equations satisfied by $F^{k}(m, \varepsilon)$, but the system will turn out to be a system of second order equations, which is difficult to handle. So we will find an equivalent system of $2 n$ differential equations which is satisfied by the $2 n$-dimensional vector

$$
\left(\begin{array}{l}
F^{k}(m, \varepsilon) \\
F^{k}(m-1, \varepsilon)
\end{array}\right)
$$

Proposition 2. we have

$$
\varepsilon \frac{d}{d \varepsilon} F^{k}(m, \varepsilon)=\mu_{k} \varepsilon F^{k}(m-1, \varepsilon)+\lambda_{k} \varepsilon^{2} F^{k}(m-2, \varepsilon)
$$


and

$$
\begin{aligned}
& \varepsilon \frac{d}{d \varepsilon} F^{k}(m, \varepsilon)=\left(\rho+m-A_{0}\right) F^{k}(m, \varepsilon)+ \\
& \quad\left(\mu_{k}-A_{1}\right) F^{k}(m-1, \varepsilon)+\left(\lambda_{k}-A_{2}\right) F^{k}(m-2, \varepsilon)
\end{aligned}
$$

(Proof.) Use (8) to have (11) and use (7) to have (12).

Writing down (11) with $m$ replaced by $m-1$, we have

$$
\begin{aligned}
\varepsilon \frac{d}{d \varepsilon}\left(\begin{array}{l}
F^{k}(m, \varepsilon) \\
F^{k}(m-1, \varepsilon)
\end{array}\right)= & \left(\begin{array}{cc}
0 & \mu_{k} \varepsilon \\
0 & 0
\end{array}\right)\left(\begin{array}{l}
F^{k}(m, \varepsilon) \\
F^{k}(m-1, \varepsilon)
\end{array}\right) \\
& +\left(\begin{array}{cc}
\lambda_{k} \varepsilon^{2} & 0 \\
\mu_{k} \varepsilon & \lambda_{k} \varepsilon^{2}
\end{array}\right)\left(\begin{array}{l}
F^{k}(m-2, \varepsilon \\
F^{k}(m-3, \varepsilon
\end{array}\right)
\end{aligned}
$$

Similarly, by using (12), we have

$$
\begin{array}{r}
\varepsilon \frac{d}{d \varepsilon}\left(\begin{array}{l}
F^{k}(m, \varepsilon) \\
F^{k}(m-1, \varepsilon)
\end{array}\right)=\left(\begin{array}{rr}
\rho+m-A_{0} & \mu_{k}-A_{1} \\
0 & \rho+m-1-A_{0}
\end{array}\right)\left(\begin{array}{l}
F^{k}(m, \varepsilon) \\
F^{k}(m-1, \varepsilon)
\end{array}\right) \\
+\left(\begin{array}{cc}
\lambda_{k}-A_{2} & 0 \\
\mu_{k}-A_{1} & \lambda_{k}-A_{2}
\end{array}\right)\left(\begin{array}{l}
F^{k}(m-2, \varepsilon) \\
F^{k}(m-3, \varepsilon)
\end{array}\right)
\end{array}
$$

To have a system of $2 n$ differential equations, we have to eliminate the vector

$$
\left(\begin{array}{l}
F^{k}(m-2, \varepsilon) \\
F^{k}(m-3, \varepsilon)
\end{array}\right)
$$

We first subtract the equation (13) from the equation (14), and substitute the resultant expression for the second vector in (13), to obtain the desired system for the first vector.

Proposition 3. $\varepsilon=0$ is a regular singular point of the system

$$
\begin{aligned}
& \varepsilon \frac{d}{d \varepsilon}\left(\begin{array}{l}
F^{k}(m, \varepsilon) \\
F^{k}(m-1, \varepsilon)
\end{array}\right)=\left(\begin{array}{rr}
0 & \mu_{k} \varepsilon \\
0 & 0
\end{array}\right)+\left(\begin{array}{rr}
\lambda_{k} \varepsilon^{2} & 0 \\
\mu_{k} \varepsilon & \lambda_{k} \varepsilon^{2}
\end{array}\right) \\
&\left(\begin{array}{ll}
\lambda_{k} \varepsilon^{2}-\lambda_{k}+A_{2} & 0 \\
\mu_{k} \varepsilon-\mu_{k}+A_{1} & \lambda_{k} \varepsilon^{2}-\lambda_{k}+A_{2}
\end{array}\right)^{-1}\left(\begin{array}{rr}
\rho+m-A_{0} & \mu_{k}-A_{1}-\mu_{k} \varepsilon \\
0 & \rho+m-1-A_{0}
\end{array}\right) \\
& \cdot\left(\begin{array}{l}
F^{k}(m, \varepsilon) \\
F^{k}(m-1, \varepsilon)
\end{array}\right)
\end{aligned}
$$

(Proof.) It is sufficient to show that the matrix

$$
\left(\begin{array}{cc}
\lambda_{k} \varepsilon^{2} & 0 \\
\mu_{k} \varepsilon & \lambda_{k} \varepsilon^{2}
\end{array}\right)\left(\begin{array}{cc}
\lambda_{k} \varepsilon^{2}-\lambda_{k}+A_{2} & 0 \\
\mu_{k} \varepsilon^{2}-A_{1} & \lambda_{k} \varepsilon^{2}-\lambda_{k}+A_{2}
\end{array}\right)^{-1}
$$


$\lambda_{k} \varepsilon^{2}-\lambda_{k}+A_{2}$ is holomorphic at the origin. We remark here that the matrix is a diagonal matrix and the inverse of $\left(\begin{array}{ll}P & O \\ Q & P\end{array}\right)$ is $\left(\begin{array}{lr}P^{-1} & 0 \\ -P^{-1} Q P^{-1} P^{-1}\end{array}\right)$.

We will carry out the proof only for $n=2$. The proof for any number of dependent variables can be done similarly. To fix the idea, we set $k=1$, and denote $d(\varepsilon)=\left(\lambda_{1} \varepsilon^{2}-\lambda_{1}+\lambda_{2}\right)$.

Now we can write down the above matrix explicitly as follows

$$
\left(\begin{array}{cccc}
\lambda_{1} \varepsilon^{2} & 0 & 0 & 0 \\
0 & \lambda_{1} \varepsilon^{2} & 0 & 0 \\
\mu_{1} \varepsilon & 0 & \lambda_{1} \varepsilon^{2} & 0 \\
0 & \mu_{1} \varepsilon & 0 & \lambda_{1} \varepsilon^{2}
\end{array}\right)\left(\begin{array}{cccc}
\lambda_{1} \varepsilon^{2} & 0 & 0 & 0 \\
0 & d(\varepsilon) & 0 & 0 \\
\mu_{1} \varepsilon & a_{12} & \lambda_{1} \varepsilon^{2} & 0 \\
a_{21} & \mu_{1} \varepsilon-\mu_{1}+a_{22} & 0 & d(\varepsilon)
\end{array}\right)
$$

where we have set

$$
A_{1}=\left(\begin{array}{ll}
a_{11} & a_{12} \\
a_{21} & a_{22}
\end{array}\right), \quad A_{2}=\left(\begin{array}{cc}
\lambda_{1} & 0 \\
0 & \lambda_{2}
\end{array}\right) .
$$

Thus we have

$$
\left(\begin{array}{cccc}
1 & 0 & 0 & 0 \\
0 & \lambda_{1} \varepsilon^{2} / d(\varepsilon) & 0 & 0 \\
\mu_{1} /\left(\lambda_{1} \varepsilon\right)-\mu_{1} \lambda_{1} \varepsilon^{2} /\left(\lambda_{1}^{2} \varepsilon^{3}\right) & -a_{12} / d(\varepsilon) & 1 & 0 \\
-a_{21} / d(\varepsilon) & \mu_{1} \varepsilon / d(\varepsilon)-\lambda_{1} \varepsilon^{2}\left(\mu_{1} \varepsilon-\mu_{1}+a_{22}\right) / d(\varepsilon)^{2} & 0 & \lambda_{1} \varepsilon^{2} / d(\varepsilon)
\end{array}\right)
$$

This shows that the matrix is holomorphic at the origin.

In general, the highest singularity that can occur is in the $(n+k, k)$ element, however, here the choice of $\mu_{k}$ makes this term disappear.

Theorem.* There are $2 n$ convergent solutions of the form (9) of the system (1).

(Proof.) The only possible singularities of the system (15) are the roots of

$$
\operatorname{det}\left(\lambda_{k} \varepsilon^{2}-\lambda_{k}+A_{2}\right)=0
$$

\footnotetext{
*) The method of convergence proof is due originally to H. Poincaré [3].
} 
Thus the part of the vector solution of (15), namely $F^{k}(m, \varepsilon)$, converges at least at $\varepsilon=1$, because of the pentagonal condition (3), and we constructed $F^{k}(m, \varepsilon)$ so that $F^{k}(m, 1)=G^{k}(m)$. Moreover there are two independent solutions of the equation (8), so that we have $2 n$ convergent solutions.

\section{REFERENCES}

[1] Kenjiro Okubo, A global representation of a fundamental set of solutions of a system of linear ordinary differential equations, J. Math. Soc. Japan, vol. 39 1963, 268-288.

[2] Kenjiro Okubo, A connection problem involving a logarithmic function. Publications of the Research Institute for Mathematical Sciences, Kyoto, University Series A, vol. $\mathbb{1} 1965,99-128$.

[3] Henri Poincaré, Sur les équations linéaires aux différentielles ordinaires et aux différences finies, Amer. J. Math. vol. 7 1885, 1-56. 